\title{
Association Between Birth Circumstances and Prenatal Maternal Mental Health - A Retrospective Study of Intergenerational Transmission
}

Vahideh MoghaddamHosseini

Sabzevar University of Medical Sciences

Neda Mahdavifar

Sabzevar University of Medical Sciences

Alexandra Makai ( $\square$ alexandra.makai@etk.pte.hu )

University of Pecs

Katalin Varga

Eötvös Loránd University

Annick Bogaerts

KU Leuven

Ákos Várnagy

University of Pecs

\section{Research Article}

Keywords: Anxiety, Breast feeding, Depression, Fear of childbirth/ W-DEQ A, Oxytocin

Posted Date: February 2nd, 2021

DOI: https://doi.org/10.21203/rs.3.rs-150994/v1

License: (1) This work is licensed under a Creative Commons Attribution 4.0 International License.

Read Full License 


\section{Abstract}

Background:The evidence on long-lasting effects of certain specific aspects of birth circumstances on the maternal mental health later during pregnancy in offspring is scares.

Aim: This study aimed to investigate the association between birth circumstances and prenatal maternal mental health in offspring.

Method: In this retrospective survey, 380 pregnant women completed the Hungarian translation of Wijma Delivery Expectancy/Experience Questionnaire A, Beck Depression Inventory-Short Form, Beck Anxiety Inventory to measure prenatal fear of childbirth (FOC), depression, and anxiety, respectively. Information on peri and postnatal events were obtained from participants' biological mothers through Mother's Birth Circumstances Questionnaire. This included questions regarding mode of birth, administered medical interventions during labor, early life care, and breastfeeding during infancy. Multiple Linear regression was performed for statistical analysis.

Results: After adjusting for potencial confounders, administration of Oxytocin (OT) induction during labor was significantly associated with higher levels of prenatal FOC $(\beta=0.14 ; 95 \% \mathrm{Cl}, 0.59,14.70)$, depression $(\beta=0.18 ; 95 \% \mathrm{Cl}, 0.47,2.73)$, and anxiety $(\beta=0.15 ; 95 \% \mathrm{Cl}, 0.50,6.95)$. Moreover, being breast fed for more than 12 months was significantly associated with lower level of prenatal FOC in offspring $(\beta=-0.12 ; 95 \%$ $\mathrm{Cl},-18.42,-1.36)$.

Conclusion: Poor prenatal mental health might be rooted in administered OT induction as a common medical practice during labor while long duration of breast feeding can have a positive effect on improving prenatal maternal mental health in offspring. Further studies of prospective design are on demand to explore the biological trajectories of these findings in humans.

\section{Introduction}

Recently there is an increasing research interest in exploring the long-term effects of birth circumstances including peri and postnatal events on offspring's mental health across the lifespan. Allen et al (1998) defined perinatal events as surgical delivery and obstetrical complications, and categorized postnatal events under two groups of early neonatal environment such as prematurity, low birth weight and anoxia, and late neonatal environment like breast feeding and infant health during the first year of life(1). In this regard, several studies have documented a significant association of low birth weight with anxiety disorders and depressive symptoms in adulthood(2,3). Also, breastfeeding during infancy, as a factor in late neonatal environment, was found to be a significant predictor for lower levels of depression, anxiety, and other mental problems in young adults $(4,5)$. In contrast, some others reported no any significant link between low birth weight and breastfeeding with mental health problems later in adolescents(6).

Despite of these informative findings, there is no any evidence on the long-term effects of other aspects of birth circumstances such as medical interventions during labor and also early life care on the maternal 
mental health during pregnancy in offspring. Maternal fear of childbirth (FOC), depression, and anxiety are relatively common mental health problems during pregnancy with the global estimated prevalence of $11.1 \%$ (7), $11.9 \%$ (8), and $15.2 \%$ (9), respectively. Prenatal maternal depression and anxiety have been frequently reported to be strong predictors for a wide range of deleterious maternal and neonatal outcomes such as lack of receiving adequate prenatal care, poor nutrition, sleep disorders, increased risk of alcohol and drugs use, suicide and infanticide, increased rate of low birth weight and preterm birth, poor maternal bonding and attachment, and certain psychological disorders in childhood and adulthood $(10,11)$. Because of the high importance of prenatal maternal mental health, it has been recently considered as one of the essential patient safety factors by the Council on Patient Safety in Women's Health Care(12).

Although a wide range of socio-demographic, obstetric, and psychological factors have been listed as contributors to prenatal FOC(13), depression(14), and anxiety(15), there is a gap in the in-depth understanding of the root of these prenatal maternal psychological problems with respect to the intergenerational transmission. The evidence on intergenerational transmission of maternal behaviors to the rearing environment that probably via epigenetic processes (such as DNA methylation) has been displayed by several studies in rats (16-18). Consistent results from primate and rodent studies confirm strongly effectiveness of the maternal environment on offspring phenotype and it's mediating by means of changes in gene expression $(16,19)$. Nevertheless, the evidence on humans is scares. Therefore, we aimed to investigate the association of birth circumstances including peri and postnatal events with maternal FOC, depression and anxiety as the most common prenatal mental health problems in offspring.

Our aim was to contribute to the already existing knowledge about the long-lasting or even transgenerational effects of maternity care received by mothers on their offspring to ameliorate prenatal maternal mental health as one of the patient safety issues.

\section{Methods}

\section{Recruitment and procedure}

A population-based cross-sectional study of healthy pregnant women was carried out from April 2016 to February 2017. Recruitment of a convenience sample of women in the third trimester of a singleton pregnancy took place at the Non-Stress Test laboratory of the Department of Obstetrics and Gynecology, Hungary.

Inclusion criteria were to be able to speak and read Hungarian, and to have their mother within reach. Women who had a history of drug abuse and/or were suffering from any major psychiatric disorder were excluded from the study.

Of the 400 pregnant women invited, 380 agreed to participate after having given informed and written consent. They were then given a booklet of questionnaires and asked to return it at the subsequent check- 
up one week later. Information on the birth circumstances was obtained from participants' biological mothers.

\section{Measurements}

The socio-demographic and obstetric checklist included questions about participntas'age, educational level, occupational status, any economic hardship, marital status, place of residence, number of pregnancies, gestational age, history of miscarriage(s), whether this pregnancy was a result of previouse planning or not.

\section{Peri and postnatal events}

Mother's Birth Circumstances Questioner(MBCQ) was mapped by questions about the date of birth (due date, preterm or postterm), mode of birth (vaginal or cesarean section), interventions during labor (administration of oxytocin, amniotomy, epidural analgesia, instrumental delivery, and episiotomy), the early life care of infants (the presence of their father during their birth, immediate skin-to-skin contact with their mother, having been breastfed during the first few hours, early rooming-in), and late life care of infants ( having been breastfed over the first two years).

\section{Prenatal Mental Health}

The Humgarien Version of Wijma Delivery Expectancy/Experience Questionnaire (W-DEQ A) that consists of 30 items was used to measure prenatal fear of childbirth. All items of this instrument are assessed on a six-point Likert scale with a range score of 0 for 'not at all' to 5 for 'extremely', according to the degree of agreement, ranging a total score from 0 to 150 (20). The Cronbach alpha in the present study accounted for 0.88 .

- Beck Anxiety Inventory (BAI) consists of 21 items was used to measure prenatal anxeity. Each item is measured on a four-point likert scale ranging from 0 ("not at all") to 3 ("severely"). The total possible score for this instrument ranges between 0 (indicating low level of anxiety ) and 63 (an indication of a high level of anxiety) (21). The Cronbach alpha in the present study accounted for 0.89 .

- Beck Depression Inventory-Short Form (BDI-SF) consists of 9 items was used to measure prenatal depression.. Each item is assessed on a four-point likert scale (from 0 to 3 ), with a total possible score ranging from 0 to 27 . The Cronbach alpha for this study tool was 0.71 .

\section{Potential confounding variable}


- Multidimensional Scale of Perceived Social Support (MSPSS) consists of 12 items was used to measure social support as a potential confounder. This instrument measures both the perceived availability and adequacy of emotional and instrumental social support from three sources of support, including family, friends, and significant others. Each item is assessed on a seven-point scale, ranging from 1 (very strongly disagree) to 7 (very strongly agree) (22). In this study, the Cronbach alpha of .86 was found for total score.

\section{Ethical statements:}

The present study was the secondary analysis of participants data recruited for the Ph.D. thesis project of the corresponding author approved by The Regional and Institutional Committee of Science and Research Ethics (No. 5923). The research was performed in accordance with the Declaration of Helsinki.

\section{Statistical analysis}

Descriptive statistics was performed to describe socio-demographic and obstetric features of participants, and psychological outcomes. Firstly, we employed Univariate linear regression model to define the association of birth circumstances with each outcome namely prenatal fear of childbirth, depression, and anxiety, independently. After that, multivariate regression models were performed to adjust obtained models for maternal age, maternal education, parity, economic status, and social support as potential confounders using stepwise method.

All estimates were reported with $95 \%$ confidence intervals $(95 \% \mathrm{Cl})$ and statistical significance was considered at the level of $P<0.05$. IBM SPSS ${ }^{\circledR}$ version 22.0 was used for the analyses.

\section{Results}

Of the 380 women who agreed to participate in the study, 371 completed and returned the questionnaires. Ten participants were excluded as they did not respond to more than half of the main items. Therefore, data of 361 participants were subject to analysis.

\section{Basic socio-demographic and obstetric characteristics}

Basic socio-demographic and obstetric characteristics of the respondents are shown in Table 1. Mean age of the participants was slightly above 32 years. Over half of the women had a high educational level and the majority of them (80.7\%) were full-time employees. Married participants accounted for almost two-thirds of the sample.

In terms of obstetric characteristics, mean gestational age of the respondents was almost 37 weeks Almost $60 \%$ of the women were multiparous and four-fifths of these women were expecting their second or third child (data not shown in Table 1). The pregnancy was unplanned in $11.5 \%$ of the cases. Almost one-third of the women reported any history of pregnancy loss until the $24^{\text {th }}$ gestational week. 


\section{Descriptive analysis of the Mother's Birth Circumstances}

Two-thirds of the respondents were born due date, and the majority of them (85.8\%) were born vaginally, according to their mothers' recollections. At least $59 \%$ of the participants' mothers had an episiotomy and a minimum of $5,7 \%$ of them were born by means of instrumental delivery (forceps/vacuum). The incidence of the administration of EDA and OT during their birth was at least $20 \%$ and $12 \%$, respectively.

Immediate skin-to-skin contact, early breastfeeding, and rooming-in took place in a minimum of $15 \%$, $24 \%$, and $13 \%$ of the cases, respectively. In addition, slightly more than half of women had been breastfed for less than six months. Table 2 illustrates all details.

\section{Descriptive analysis of Prenatal Fear of Childbirth, Depression, Anxiety}

Mean scores (SD) on scales W-DEQ A, BDI-SF, and BAI was obtained 52.43(20.58), 10.91(9.30), and $4.25(3.61)$, respectively.

\section{The Association Between Mother's birth Circumstances and Prenatal Mental Health}

After adjusting the regression models for maternal age, maternal education, parity, economic status, and perceived social support as potential confounders, the findings revealed a significant association between administration of Oxytocin induction during labor and higher levels of prenatal fear of childbirth $(\beta=0.14 ; 95 \% \mathrm{Cl}, 0.59,14.70)$, depression $(\beta=0.18 ; 95 \% \mathrm{Cl}, 0.47,2.73)$, and anxiety $(\beta=0.15 ; 95 \% \mathrm{Cl}, 0.50$, 6.95). Moreover, being breast fed for more than 12 months was significantly associated with the lower level of prenatal fear of childbirth in offspring $(\beta=-0.12 ; 95 \% \mathrm{Cl},-18.42,-1.36)$.

Neither all the other medical interventions during labor, nor the early life care factors, was significantly linked with the prenatal mental health before and after controlling for confounders (Table 3 ).

\section{Discussion}

This study aims to be the first one to map the link between peri and postnatal events and offspring's prenatal fear of childbirth, depression, and anxiety as the most common maternal psychological problems during pregnancy with regard to the intergenerational transmission. Our results revealed that the administration of OT induction is significantly associated with higher levels of fear of childbirth, depression, and anxiety later during pregnancy in offspring. Moreover, while, there was a significant relationship between being breast fed for more than 12 months and lower level of fear of childbirth, this result was not obtained for prenatal depression and anxiety. 


\section{Hormonal plausibility}

The trajectory that could justify our findings is related to an anxiolytic feature of endogenous OT in the reduction of maternal fear and anxiety. Increasing number of human and animal studies $(23,24)$ support the role of endogenous OT in attenuating fear-related anxiety through inhibition of the stress-induced activation of the HPA axis and the reduction of the amygdala activity (25). Infusing OT receptor antagonists into the central amygdala resulted in an anxiogenic behavior in pregnant rats (26). Certain studies called the attention to the possible fetal effects of exogenous OT administered in the perinatal period. A decreased amount of face-to-face contact between mother and newborn in the first hour postpartum (27), weaker neonatal sucking reflexes (28), and interrupted pre-feeding (29) were observed in connection with labor induction by administration of exogenous OT. Syntactic OT administered during labor is able to alter physiological and emotional regulation in the mother that can influence the newborn indirectly via epigenetically transmission from mother to offspring (30). Given that in our study, the mother's recollections of OT administration during labor was significantly associated with poor prenatal mental health in their offspring, a tentative long-term effect of the insufficient level of endogenous OT and/or OT receptor on the offspring's psychological and emotional development could be concluded.

The association of breast feeding with lower levels of fear of childbirth could be interpreted through the level of OT in lactating women and also developing greater maternal bonding and attachment style in offspring. the higher levels of OT and consequently, more positive mood and less aggression and depression in lactating women in comparison with non-lactating ones have been well documented across studies(31). Furthermore, the longer duration of breast feeding plays a known role in forming bonding and secure attachment style. On the other hand, a correlation between self-report of attachment style and the levels of plasma OT has been observed, demonstrating the higher levels of OT in women with secure attachment with their parents $(32,33)$. The increased level of OT during the postnatal period can be transmitted to the following generation and have positive impact on them. Recently, Porta et al. (2016) pointed to the intergenerational transmission of breastfeeding behavior. It possibly happens through epigenetic mechanisms that can affect the offspring's OT receptor signaling(34). In consistent with our findings, a longitudinal prospective study showed a significant prediction of disruptive behavior in adulthood at age 30 by breastfeeding during infancy. However, family support was a significant mediator for this relationship(35). Simirarly, social support remained significantly in our final model, as well.

In relation to early life care, we did not gain significant results. In contrast, the positive impact of early contact between mother and newborn (such as skin-to-skin, eye contact, touching and verbal cues) in the activation of the OT system has been documented (36). Stolzenberg and Champagne (2016) pointed to the experience-induced basis of maternal behavior which similarly to hormone-induced pathways might be able to lead to epigenetic changes via chromatin remodeling. This can play a significant role in the initiation and continuation of the mother-infant interactions (37).

A good deal of attention should be given to the role of other hormones (such as cortisol, estrogen, progesterone, endogenous opioids) and their receptor systems in maternal neuropsychological health 
and their interaction with OT (38). Furthermore, basic individual differences, and previous and current maternal experiences should be taken into account.

\section{Strength and limitation}

Considering the mother's birth circumstances in the examination of intergenerational transmission of an effective component of reproductive behavior in human, likely through an epigenetic mechanism brings a principle novelty in this area, presenting a new scientific approach for future studies.

One limitation of the study is that certain obstetric practices such as EDA, skin-to-skin contact with mother, rooming-in, and father's presence at birth were not common at the time when participants were born. Therefore, the rate of negative answers to these items was relatively high, resulting in a nonsignificant relationship. Also, fading memories of childbirth circumstances might have led to recall bias. Nevertheless, certain studies have noted that maternal recall of some peri and postnatal events, especially regarding initiation and duration of breast feeding, have acceptable accuracy(39).

\section{Conclusion}

This study, after adjusting for potential confounders, revealed that administration of OT induction can significantly predict poor prenatal maternal mental health while, breast feeding for more than 12 months was a significant predictor for the lower level of fear of childbirth, resulting in better maternal mental health later during pregnancy in offspring. Our findings support this theory that poor prenatal maternal mental health might be rooted in perinatal events and late neonatal care, showing the high importance of attention from maternity care givers to long-lasting effects of perinatal and postnatal events. This effect might be mediated through the tentative role of plasma OT and/or OT receptors in mothers and its capability of being transmitted to the next generation and having a negative or positive effect on them.

Further studies of prospective design are on demand to explore the precise biological trajectories and molecular background of these findings in humans.

\section{Declarations}

Disclosure of interests: The authors declare that they have no any conflicts of interest.

\section{Author Contribution}

Vahideh MoghaddamHosseini designed and performed study, and wrote the manuscript. Katalin Varga and Ákos Várnagy supervised the study and revised the manuscript. Alexandra Makai and Neda Mahdavifar conducted statistical analysis. Annick Bogaerts revised the manuscript

All authors contributed to and have approved the final manuscript. 


\section{References}

1. Allen, N. B., Lewinsohn, P. M. \& Seeley, J. R. Prenatal and perinatal influences on risk for psychopathology in childhood and adolescence. Dev Psychopathol.10 (3), 513-529 (1998).

2. Alati, R. et al. Fetal growth and behaviour problems in early adolescence: findings from the Mater University Study of Pregnancy. Int J Epidemiol.38 (5), 1390-1400 (2009).

3. Nomura, Y. et al. Low birth weight and risk of affective disorders and selected medical illness in offspring at high and low risk for depression. Compr Psychiatry.48 (5), 470-478 (2007).

4. de Mola, C. L. et al. Breastfeeding and mental health in adulthood: A birth cohort study in Brazil. $J$ Affect Disord.202, 115-119 (2016).

5. Hayatbakhsh, M. R., O'Callaghan, M. J., Bor, W., Williams, G. M. \& Najman, J. M. Association of breastfeeding and adolescents' psychopathology: a large prospective study. Breastfeed Med.7 (6), 480-486 (2012).

6. Wiles, N. J. et al. Fetal growth and childhood behavioral problems: results from the ALSPAC cohort. Am J Epidemiol.163 (9), 829-837 (2006).

7. Nilsson, C. et al. Definitions, measurements and prevalence of fear of childbirth: a systematic review. BMC Pregnancy Childbirth.18 (1), 28 (2018).

8. Woody, C. A., Ferrari, A. J., Siskind, D. J., Whiteford, H. A. \& Harris, M. G. A systematic review and meta-regression of the prevalence and incidence of perinatal depression. J Affect Disord.219, 86-92 (2017).

9. Dennis, C-L., Falah-Hassani, K. \& Shiri, R. Prevalence of antenatal and postnatal anxiety: systematic review and meta-analysis. Br J Psychiatry.210 (5), 315-323 (2017).

10. Leight, K. L., Fitelson, E. M., Weston, C. A. \& Wisner, K. L. Childbirth and mental disorders. Int Rev Psychiatry.22 (5), 453-471 (2010).

11. Schetter, C. D. \& Tanner, L. Anxiety, depression and stress in pregnancy: implications for mothers, children, research, and practice. Curr Opin Psychiatry. 25 (2), 141 (2012).

12. Kendig, S. et al. Consensus bundle on maternal mental health: perinatal depression and anxiety. $J$ Obstet Gynecol Neonatal Nurs.46 (2), 272-281 (2017).

13. Dencker, A. et al. Causes and outcomes in studies of fear of childbirth: a systematic review. Women Birth.32 (2), 99-111 (2019).

14. Gelaye, B., Rondon, M. B., Araya, R. \& Williams, M. A. Epidemiology of maternal depression, risk factors, and child outcomes in low-income and middle-income countries. Lancet Psychiatry.3 (10), 973-982 (2016).

15. Leach, L. S., Poyser, C. \& Fairweather-Schmidt, K. Maternal perinatal anxiety: A review of prevalence and correlates. Clin Psychol.21 (1), 4-19 (2017).

16. Champagne, F. A. Epigenetic mechanisms and the transgenerational effects of maternal care. Front Neuroendocrinol.29 (3), 386-397 (2008). 
17. Champagne, F., Diorio, J., Sharma, S. \& Meaney, M. J. Naturally occurring variations in maternal behavior in the rat are associated with differences in estrogen-inducible central oxytocin receptors. Proc Natl Acad Sci.98 (22), 12736-12741 (2001).

18. Francis, D. D., Young, L. J., Meaney, M. J. \& Insel, T. R. Naturally occurring differences in maternal care are associated with the expression of oxytocin and vasopressin (V1a) receptors: gender differences. J Neuroendocrinol.14 (5), 349-353 (2002).

19. Lovic, V., Gonzalez, A. \& Fleming, A. S. Maternally separated rats show deficits in maternal care in adulthood. Dev Psychobiol.39 (1), 19-33 (2001).

20. MoghaddamHosseini, V., Makai, A., Dweik, D. \& Várnagy, Ã. Factor analysis study of the Hungarian translation of Wijma Delivery Expectancy/Experience Questionnaire (version A).Curr Psychol. 2018;1-8.

21. Beek, A. T., Epstein, N., Brown, G. \& Steer, R. A. An inventory for measuring clinical anxiety: Psychometrie properties. J OfConsulting Clin Psychol.56 (6), 893-897 (1988).

22. Zimet, G. D., Powell, S. S., Farley, G. K., Werkman, S. \& Berkoff, K. A. Psychometric characteristics of the multidimensional scale of perceived social support. J Pers Assess.55 (3-4), 610-617 (1990).

23. Mezzacappa, E. S. \& Katkin, E. S. Breast-feeding is associated with reduced perceived stress and negative mood in mothers. Health Psychol.21 (2), 187 (2002).

24. Lahoud, N. \& Maroun, M. Oxytocinergic manipulations in corticolimbic circuit differentially affect fear acquisition and extinction. Psychoneuroendocrinology.38 (10), 2184-2195 (2013).

25. Lee, H-J., Macbeth, A. H., Pagani, J. H. \& Young, W. S. Oxytocin: the great facilitator of life. Prog Neurobiol.88 (2), 127-151 (2009).

26. Neumann, I. D., Krömer, S. A., Toschi, N. \& Ebner, K. Brain oxytocin inhibits the (re) activity of the hypothalamo-pituitary-adrenal axis in male rats: involvement of hypothalamic and limbic brain regions. Regul Pept.96 (1), 31-38 (2000).

27. Trevathan, W. R. Maternal "en face" orientation during the first hour after birth. Am J Orthopsychiatry.53 (1), 92 (1983).

28. Olza Fernández, I. et al. Newborn feeding behaviour depressed by intrapartum oxytocin: a pilot study. Acta Paediatr.101 (7), 749-754 (2012).

29. Bell, A. F., White-Traut, R. \& Rankin, K. Fetal exposure to synthetic oxytocin and the relationship with prefeeding cues within one hour postbirth. Early Hum Dev.89 (3), 137-143 (2013).

30. Kenkel, W. M., Yee, J. R. \& Carter, C. S. Is oxytocin a maternal-foetal signalling molecule at birth? Implications for development. J Neuroendocrinol.26 (10), 739-749 (2014).

31. Uvnäs-Moberg, K. Role of efferent and afferent vagal nerve activity during reproduction: integrating function of oxytocin on metabolism and behaviour. Psychoneuroendocrinology.19 (5-7), 687-695 (1994).

32. Strathearn, L., Fonagy, P., Amico, J. \& Montague, P. R. Adult attachment predicts maternal brain and oxytocin response to infant cues. Neuropsychopharmacology.34 (13), 2655-2666 (2009). 
33. Feldman, R. et al. Sensitive parenting is associated with plasma oxytocin and polymorphisms in the OXTR and CD38 genes. Biol Psychiatry.72 (3), 175-181 (2012).

34. Porta, F. et al. Genealogy of breastfeeding. Eur J Pediatr.175 (1), 105-112 (2016).

35. Essau, C. A., Sasagawa, S., Lewinsohn, P. M. \& Rohde, P. The impact of pre-and perinatal factors on psychopathology in adulthood. J Affect Disord.236, 52-59 (2018).

36. Matthiesen, A-S., Ransjö-Arvidson, A-B., Nissen, E. \& Uvnäs-Moberg, K. Postpartum maternal oxytocin release by newborns: effects of infant hand massage and sucking. Birth.28 (1), 13-19 (2001).

37. Stolzenberg, D. S. \& Champagne, F. A. Hormonal and non-hormonal bases of maternal behavior: the role of experience and epigenetic mechanisms. Horm Behav.77, 204-210 (2016).

38. Swain, J. E., Kim, P. \& Ho, S. S. Neuroendocrinology of parental response to baby-cry. J Neuroendocrinol.23 (11), 1036-1041 (2011).

39. Li, R., Scanlon, K. S. \& Serdula, M. K. The validity and reliability of maternal recall of breastfeeding practice. Nutr Rev.63 (4), 103-110 (2005).

\section{Tables}

Table 1: Participants' socio-demographic and obstetric characteristics 


\begin{tabular}{|ll|}
\hline Socio-demographic characteristics & \\
\hline Age (Mean \pm SD) & $32.41 \pm 5.32$ \\
\hline Education $n$ (\%) & \\
\hline Low & $18(5.0)$ \\
\hline Intermediate & $154(42.9)$ \\
\hline High & $187(52.1)$ \\
\hline Employment status $n$ (\%) & \\
\hline Full time employee & $298(80.7)$ \\
\hline Part time employee & $7(2.0)$ \\
\hline Self-employed & $28(7.8)$ \\
\hline Housewife & $34(9.5)$ \\
\hline Marital status $n$ (\%) & \\
\hline Married & $222(62.0)$ \\
\hline Cohabiting & $128(35.8)$ \\
\hline Single/divorced/widow & $8(2.5)$ \\
\hline Place of residence $n$ (\%) & \\
\hline Capital/county seat & $134(40.1)$ \\
\hline City & $215(59.9)$ \\
\hline Village & $134(37.5)$ \\
\hline Economic hardship $n(\%)$ & $92(25.8)$ \\
\hline Yes & \\
\hline No & \\
\hline Obstetrics characteristics & \\
\hline Gestational age (Mean \pm SD) & \\
\hline Parity $n$ (\%) & \\
\hline Nulliparous & \\
\hline Multiparous & \\
\hline Pregnancy Status $n(\%)$ & \\
\hline
\end{tabular}




\begin{tabular}{|lc|} 
Unplanned & $41(11.5)$ \\
\hline History of miscarriage(s) and/or termination(s) of pregnancy $\boldsymbol{n}(\%)$ \\
\hline Yes & $109(30.4)$ \\
\hline No & $249(69.6)$ \\
\hline
\end{tabular}

Table 2: Descriptive statistics on mother's birth circumstances (based on the recollections of their mothers) 


\begin{tabular}{|c|c|c|c|c|}
\hline Date of Birth & $n(\%)$ & & & \\
\hline Full term & & $231(64.5)$ & & \\
\hline Preterm & & $42(11.7)$ & & \\
\hline Post term & & $57(15.9)$ & & \\
\hline I do not know & & $28(7.8)$ & & \\
\hline Mode of Birth & & & & \\
\hline Vaginal delivery & & $308(85.8)$ & & \\
\hline Elective Cesarean section & & $36(10.0)$ & & \\
\hline Emergency Cesarean sectio & & $13(3.6)$ & & \\
\hline I do not know & & $2(0.6)$ & & \\
\hline Medical Interventions & & Yes & No & I do not know \\
\hline & & $n(\%)$ & $n(\%)$ & $n(\%)$ \\
\hline Episiotomy & & $182(59.1)$ & $47(15,3)$ & $79(25,6)$ \\
\hline Amniotomy & & $71(20.7)$ & $113(32.9)$ & $159(46.4)$ \\
\hline Instrumental delivery & & $19(5.7)$ & $204(61.3)$ & $110(33.0)$ \\
\hline Epidural anesthesia & & $70(20.6)$ & $200(58.9)$ & $69(20.5)$ \\
\hline Oxytocin induction & & $40(11.8)$ & $186(54.7)$ & 114(33.5) \\
\hline Early Life Care & & & & \\
\hline Father present at labor and & ivery & $22(6.3)$ & $279(80.4)$ & 46(13.3) \\
\hline Immediate skin-to-skin cont & with mother after birth & $54(15.5)$ & $129(37.1)$ & $165(47.4)$ \\
\hline Having been breastfed in th & rst few hours after birth & $83(24.1)$ & $85(24.6)$ & 177(51.3) \\
\hline Rooming-in with mother dur & the hospital stay & 46(13.3) & $167(48.4)$ & 132(38.3) \\
\hline late Life Care (breast feedin & ver the first 2 years) & & & \\
\hline Not having been breastfed & & $3(1.1)$ & & \\
\hline Having been breastfed for & less than 6 months & 138(52.3) & & \\
\hline Having been breastfed for & 6-12 months & $95(27.0)$ & & \\
\hline Having been breastfed for & more than 12 months & $28(10.6)$ & & \\
\hline
\end{tabular}

Table 3: Summary of the multiple linear regression analyses of association between mother's birth 
circumstances and prenatal mental health (only remained variables in the final model have been presented).

\begin{tabular}{|lll|}
\hline Variables & $\begin{array}{l}\text { Crude } \\
\beta \text { coefficient }(95 \% \mathrm{Cl})\end{array}$ & $\begin{array}{l}\text { Adjusted } \\
\boldsymbol{\beta} \text { coefficient }(95 \% \mathrm{Cl})\end{array}$ \\
\hline Prenatal Fear of Childbirth & & \\
\hline Administrated Oxytocin Induction & $.15(.92,15.34)^{*}$ & $.14(56,14.70)^{*}$ \\
\hline Parity & $-.22(-6.27,-2.25)^{* *}$ & $-.23(-6.43,-2.43)^{* *}$ \\
\hline Having been breast fed for more than 12 months & $-.10(-17.24, .17)^{*}$ & $-.12(-18.42,-1.37)^{*}$ \\
\hline Social Support & $-.11(-.38,-.02)^{*}$ & $-.11(-.36,-.00)^{*}$ \\
\hline High level of Education & $-.14(-5.00,-.72)^{* *}$ & $-.13(-4.71,-.43)^{*}$ \\
\hline Prenatal Depression & & \\
\hline Administrated Oxytocin Induction & $.19(50,2.73)^{* *}$ & $.18(.47,2.73)^{* *}$ \\
\hline Social Support & $-.27(-.11,-.05)^{* *}$ & $-.15(-.08,-.006)^{*}$ \\
\hline Prenatal Anxiety & & \\
\hline Administrated Oxytocin Induction & $.15(.45,6.85)^{*}$ & $.16(50,6.94)^{*}$ \\
\hline Having high economic status & $-.15(-.5 .07,-.80)^{* *}$ & $-.17(-.5 .53,-.51)^{*}$ \\
\hline
\end{tabular}

$$
\text { * } p<0.05 \quad \text { ** } p<0.01
$$

